\title{
The landscape of Blockchain research: impacts and opportunities
}

\section{Hsing Kenneth Cheng ${ }^{1}$ - Daning $\mathrm{Hu}^{2} \cdot$ Thomas Puschmann ${ }^{3} \cdot$ J. Leon Zhao ${ }^{4}$}

Published online: 7 November 2021

(c) The Author(s), under exclusive licence to Springer-Verlag GmbH Germany, part of Springer Nature 2021, corrected publication 2021

\begin{abstract}
Blockchain technology has emerged as an important research domain in recent years. It not only supports the secure and efficient storage and processing of information but may also transform the business principles and processes embedded in traditional centralized organizations and societies. This editorial first provides a framework that identifies the emerging areas of blockchain research. The key characteristics of this framework in Blockchain 1.0, 2.0, and 3.0 are defined and introduced. The impacts and opportunities associated with blockchain research are identified and discussed. At last, the six articles in this special issue are characterized using the proposed research framework of blockchain research.
\end{abstract}

\section{Introduction}

The notion of blockchain technology and its applications in various business domains have attracted a lot of attention. Although the most well-known application of blockchain technology is Bitcoin, its applications go far beyond cryptocurrency. In this editorial, we introduce six articles included in our special issue which

Daning Hu

hudn@sustech.edu.cn

Hsing Kenneth Cheng

kenny.cheng@warrington.ufl.edu

Thomas Puschmann

thomas.puschmann@bf.uzh.ch

J. Leon Zhao

leonzhao@cuhk.edu.cn

1 University of Florida, Gainesville, USA

2 Southern University of Science and Technology, Shenzhen, China

3 University of Zurich, Zürich, Switzerland

4 Chinese University of Hong Kong, Shenzhen, China 
encompass a number of important topics on blockchain technology and applications, and discuss the current status and opportunities of research in this emerging area mainly from the business perspective.

Blockchain is based upon the novel ideas of a distributed ledger, an innovative approach of decentralized storage, and secured management of large amounts of transaction data outside the boundary of a conventional organization. Its advantages include anonymity, real-time data management, decentralization, and disintermediation. With these features, blockchain revolutionizes how transaction information is stored and managed in networked markets and institutions. Currently, the majority of blockchain applications concentrated in the information technology and financial domain. But more and more blockchain applications are expanding to other important areas like supply chain and the Internet of Things.

Information Systems (IS) scholars are becoming increasingly interested in studying blockchain technology and applications. This special issue includes six studies on this topic using various research methods like design science, behavioral research, or IS economics. As Table 1 shows, previous research (Zhao et al. 2016) has suggested that the development of blockchain technology has gone through three generations, namely, Blockchain 1.0 for digital currency (cryptocurrency), Blockchain 2.0 for digital finance, and Blockchain 3.0 for digital society (Xu et al. 2019). This editorial extends on this framework to lay a foundation for these six articles and identify several promising research directions in blockchain research from a business research perspective.

\section{Blockchain 1.0}

The blockchain technologies and applications in digital currency (especially cryptocurrency) is often referred to as blockchain 1.0. Cryptocurrency has attracted a lot of attention largely because of the rise of the Bitcoin over the past ten years. Bitcoin has envisioned an innovative form of digital currency-cryptocurrency. Nowadays, the majority of global blockchain companies are related to cryptocurrency. Cryptocurrency has challenged the traditional paradigm of state-controlled currencies and the dominant role of central banks, as well as the conventional financial institutions (Dong 2018). In recent years, many central banks are also preparing to launch their own fiat digital currencies.

Given the volatile nature of existing cryptocurrencies, they were considered as a type of risky financial asset (Guesmi et al. 2019). There is a stream of research studying the cryptocurrency markets from the perspectives of market efficiency, volatility clustering, and portfolio management (Corbet et al. 2019). The hype of Bitcoin also led to a lot of research related to Blockchain technology. We conducted a search of "blockchain" in the Web of Science search engine provided by Thomson Reuters and found 3,413 published articles (As of 06-26-2020).

The first paper in our special issue "A Scientometric Review of Blockchain Research" adopted multiple scientometric methods to generate a panoramic view of the recent developments in blockchain research. It identifies four research aspects in current blockchain research: underlying technology architecture, privacy and 


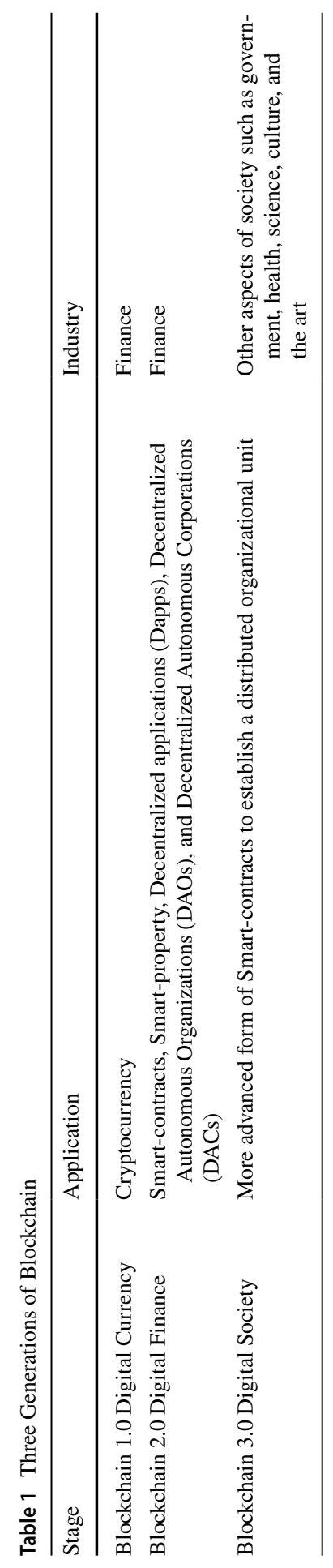


security, financial applications, and smart scene applications. Based on the results of literature detection and structural variation analysis, they suggested two important emerging trends in blockchain research: 1) solving problems caused by applying cryptocurrencies in the real world, and 2) improving blockchain technology based on specific application requirements.

The second paper " a survey of blockchain with applications in maritime and shipping industry" categorizes recent blockchain research (2015-2018) into four areas: hardware, software, emerging technology, and business applications. It examined the impacts of blockchain technology and applications on cost, quality, speed, and risk assessment in shipping and supply chain management. The authors found that the mechanisms of blockchain that can greatly improve the efficiencies of international shipping and supply chain. Lastly, the study evidenced the increasing transparency and accountability of international shipping provided by blockchain technology.

\section{Blockchain 2.0}

Blockchain 2.0 was often referred to as financial applications of blockchain besides digital currency, such as securities trading, supply chain finance, and banking instruments (Xu et al. 2019). For instance, blockchain has been transforming traditional trading methods in practice. Kim and Journal (2018) proposed that blockchain can reduce the time of key trading processes, including currency transactions and identity verifications, thereby improving efficiency in record keeping and equity trading. Similarly, Gomber et al. (2018) suggested that blockchain is a disruptive financial innovation for supporting new business models and market mechanisms. Moreover, previous studies have envisaged that blockchain technology can greatly transformed traditional banking operations through its advantages in speed, accuracy, reliability, security, and traceability (Arvind and Biot-Paquerot 2018; Mori 2016). Chen et al. (2017) found that blockchain can change the way banks communicate with customers, as well as business models and even the banking ecology.

Following this line of research, the paper "The impact of Blockchain on Business Models in Banking" studied how blockchain affect banking business models. It developed a research model that connects IT innovations with the three banking value disciplines: operational excellence, customer intimacy, and product leadership, and the four generic elements of business models: "what", "who", "how", and "value". This model was examined through surveying 104 executives of both banks and non-banks around the world. The results show that all three value disciplines are significantly affected by blockchain technology.

Another common Blockchain 2.0 application is smart contract that can automatically execute various business processes based on pre-specified concepts and rules. It has revolutionized traditional financial transaction and payment systems. Two important extensions of long-term smart contracts are decentralized autonomous organizations (DAOs) and Decentralized Autonomous Corporations (DACs), which contain the digital assets and encode the bylaws of an entire organization and corporations. These concepts are the foundation of the development and user communities under the emerging Fintech platforms. Moreover, smart contracts are traceable and 
irreversible. They allow transaction records to be maintained publicly in a secure and trustful environment (Wang et al. 2019). Current application domains of smart contracts include Decentralized finance (DeFi), supply chain management, Internet of Things, healthcare, insurance, and financial systems (Mohanta, Panda, and Jena 2018).

The paper "Laying the Foundation for Smart Contract Development: An Integrated Engineering Process Model" proposes an integrated process model for engineering Distributed Ledger Technology (DLT)-based smart contracts. This model can be iteratively refined by domain experts. It explicitly accounts for the immutability of the trustless, append-only, and decentralized DLT ecosystem, thereby overcoming certain limitations of traditional software engineering process models. The model consists of five phases: conceptualization, implementation, approval, execution, and finalization. Applying such a model of engineering smart contracts can help developers better understand and streamline the engineering process of DLTs. This model can also serve as a generic framework of supporting DLT-based application development in general.

\section{Blockchain 3.0}

The blockchain technology envisioned a new form of the economy with the core value of trust. Blockchain 3.0 is a blueprint for popularizing the technology in fields other than cryptocurrency and finance, such as government, health, science, culture, and the arts (Swan 2015). It focuses on the regulation and governance of blockchainbased decentralization in every aspect of society.

A main challenge that blockchain technology aims to tackle is to promote trust through reliable and efficient information sharing, given its characteristics such as distributed operation, authentication, the immutability of records, and cryptography. Luhmann (2018) categorized trust into two types: personal and system trust. Personal or organizational relationships and emotional bond promote the former type of trust, while consensus rules enforce the latter. The adoption of blockchain technology allows us to replace personal trust with system trust. Therefore, blockchain technology as a "truth machine" has the potential to reshape the business relationships and processes in society.

In this special issue, the paper entitled "How can we reduce information asymmetries and enhance trust in the market for lemons?" explores how blockchain can reduce information asymmetry in a used car market. They demonstrated that the blockchain-based platform helps reduce information asymmetries. Trust in the IT artifact plays a vital role as a mediator in building initial trust in the system itself. By employing the design science approach, they demonstrated how the provision of car usage data in a blockchain platform can reduce information asymmetries and enhance trust in the used car market.

Moreover, blockchain technology has many applications in supply chain management. Tracking goods through the production and delivery process requires automated compliance to freight and trade regulations. Schmidt and Wagner (2019) argued that blockchain technology limits opportunistic behavior, environmental, and 
behavioral uncertainty. Correspondingly, it could reduce transaction costs and enables more market-oriented governance structures.

The paper "Processes, Benefits, and Challenges for Adoption of Blockchain Technologies in Food Supply Chains: A Thematic Analysis" examined the processes, benefits, and challenges of adopting blockchain technologies in food supply chains. This paper adopted the thematic analysis method to identify seven first-order themes and two second-order themes in adoption processes; thirteen first-order themes, sixteen second-order themes, and five third-order themes in benefits; and fourteen first-order themes and five second-order themes in challenges. Its findings help us to better understand the benefits and challenges of adopting blockchain in food supply chains.

\section{Future research directions}

Beyond the three-type categorization of blockchain development, an emerging field that is based on blockchain technology is Decentralization Finance, often referred to as 'DeFi'. It is a novel experimental form of finance that does not rely on central authorities or intermediaries like exchanges or banks but mainly utilizes smart contracts. Technically, DeFi does not refer to a single project but an overall integration of decentralized financial services and applications that are based on blockchain technology (Abdulhakeem and Hu 2021). For example, Facebook's stable coin Diem aims at offering a global digital payment method independent from financial institutions. DeFi aims to provide a low-cost, fast, efficient, trustworthy, and completely transparent global financial ecosystem that operates over the internet without any central authority and is highly accessible to everyone around the globe (Abdulhakeem and $\mathrm{Hu} 2021$ ).

As the DeFi field is still very young compared to the aforementioned blockchain research topics, it has many possible research directions with great potential, such as stable coins, liquidity pools, decentralized exchanges, decentralized governance mechanisms, as well as unique security issues. We believe that DeFi research will attract a lot of interests in the near future.

\section{References}

Abdulhakeem SA, Hu Q (2021) Powered by Blockchain technology, DeFi (Decentralized Finance) strives to increase financial inclusion of the unbanked by reshaping the world financial system. Mod Econ 12(01):1

Arvind A, Biot-Paquerot G (2018) FinTech evolution: Strategic value management issues in a fast changing industry. Strateg Change 27(4):301-311

Ashta A, Biot-Paquerot G (2018) FinTech evolution: strategic value management issues in a fast changing industry. Strateg Chang 27(4):301-311. https://doi.org/10.1002/jsc.2203

Chen Z, Li Y, Wu Y, Luo J (2017) The transition from traditional banking to mobile internet finance: an organizational innovation perspective-a comparative study of Citibank and ICBC. Financial Innovation 3(1):1-16. https://doi.org/10.1186/s40854-017-0062-0

Corbet S, Lucey B, Urquhart A, Yarovaya L (2019) Cryptocurrencies as a financial asset: a systematic analysis. Int Rev Financ Anal 62:182-199. https://doi.org/10.1016/j.irfa.2018.09.003

Dong, H. (2018). Monetary policy in the digital age. Finance \& Development 55(2). 
Gomber P, Kauffman RJ, Parker C, Weber BW (2018) On the fintech revolution: interpreting the forces of innovation, disruption, and transformation in financial services. J Manag Inf Syst 35(1):220-265. https://doi.org/10.1080/07421222.2018.1440766

Guesmi K, Saadi S, Abid I, Ftiti Z (2019) Portfolio diversification with virtual currency: evidence from bitcoin. Int Rev Financial Anal 63:431-437. https://doi.org/10.1016/j.irfa.2018.03.004

Kim, S., \& Journal, A. S. J. S. E. (2018). Distributed Ledger and Blockchain Technology: Framework and Use Cases. Forthcoming, Journal of Investment Management.

Luhmann N (2018) Trust and power. John Wiley \& Sons

Mohanta, B. K., Panda, S. S., \& Jena, D. (2018). An overview of smart contract and use cases in blockchain technology. 2018 9th International Conference on Computing, Communication and Networking Technologies (ICCCNT). https://doi.org/10.1109/ICCCNT.2018.8494045

Mori T (2016) Financial technology: Blockchain and securities settlement. J Securities Operations \& Custody 8(3):208-227

Schmidt CG, Wagner SM (2019) Blockchain and supply chain relations: a transaction cost theory perspective. J Purch Supply Manag 25(4):100552. https://doi.org/10.1016/j.pursup.2019.100552

Swan, M. (2015). Blockchain: Blueprint for a new economy. O’Reilly Media, Inc.

Wang S, Ouyang L, Yuan Y, Ni X, Han X, Wang F-Y (2019) Blockchain-enabled smart contracts: architecture, applications, and future trends. IEEE Trans Syst Man, and Cybernet: Syst 49(11):22662277. https://doi.org/10.1109/TSMC.2019.2895123

Xu M, Chen X, Kou G (2019) A systematic review of blockchain. Financial Innovation 5(1):27. https:// doi.org/10.1186/s40854-019-0147-z

Zhao JL, Fan S, Yan J (2016) Overview of business innovations and research opportunities in blockchain and introduction to the special issue. Financial Innovation 2:28. https://doi.org/10.1186/ s40854-016-0049-2

Publisher's Note Springer Nature remains neutral with regard to jurisdictional claims in published maps and institutional affiliations. 